\title{
Extracellular Vesicles in Regenerative Medicine, a Brief Review
}

\author{
Mehdi Soleymani-Goloujeh ${ }^{1}$, Samaneh Saberi² and Faezeh Shekari ${ }^{3}$ 4* $^{*}$ \\ 1. Dept. of Stem Cells and Developmental Biology at Cell Science Research Center, Royan Institute for Stem Cell Biology and \\ Technology, ACECR, Tehran, Iran \\ 2. HPGC Research Group, Department of Medical Biotechnology, Pasteur Institute of Iran, Tehran, Iran \\ 3. Dept. of Molecular Systems Biology, Cell Science Research Center, Royan Institute for Stem Cell Biology and Technology, \\ ACECR, Tehran, Iran \\ 4. Dept. of Developmental Biology, University of Science and Culture, Tehran, Iran
}

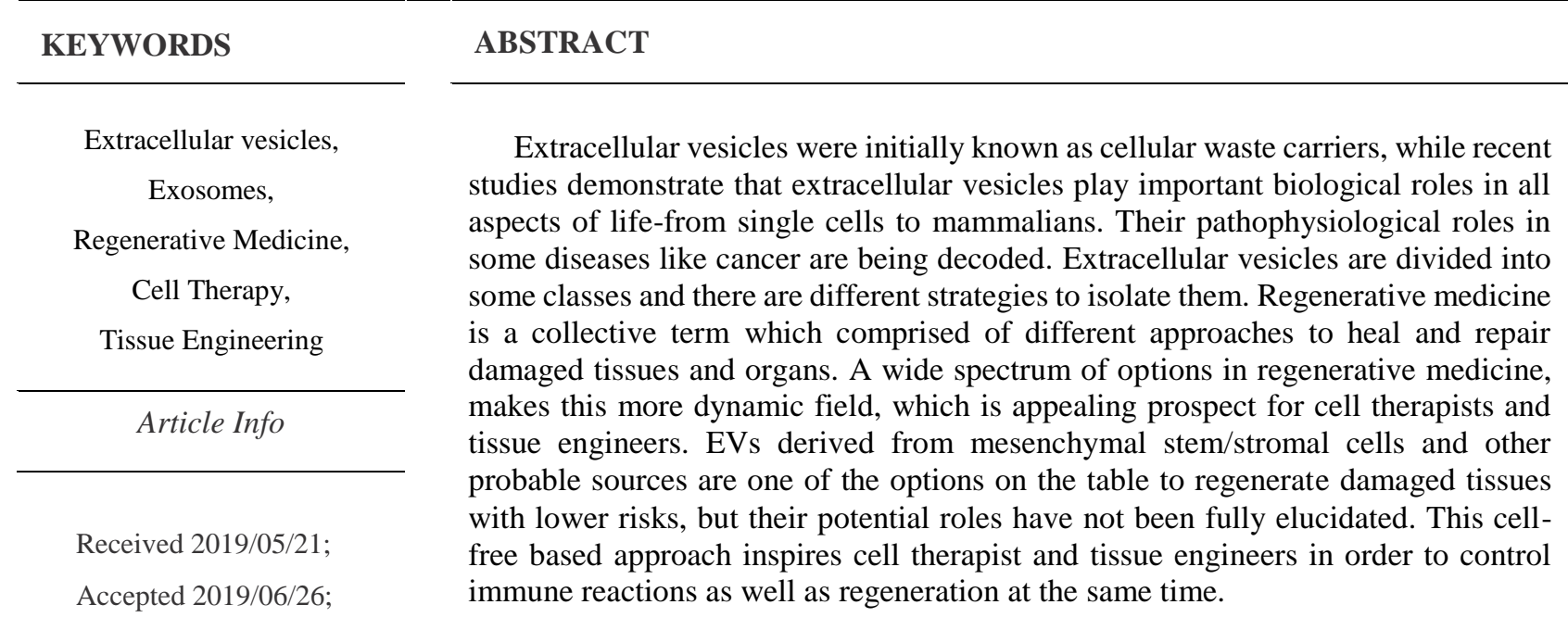

Published Online 2019

Corresponding Information: Faezeh Shekari Ph.D., Royan Institute, Banihashem Sq., Banihashem St., Ressalat Highway, Tehran, Iran E-mail: Faezehshekari@gmail.com

Copyright $\odot$ (2019. This is an open-access article distributed under the terms of the Creative Commons Attribution-noncommercial 4.0 International License which permits copy and redistribute the material just in noncommercial usages, provided the original work is properly cited.

\section{Introduction}

Nowadays, it has been demonstrated that cells from different organisms, from eukaryotes to prokaryotes can release vesicular bodies into the extracellular environment $(1,2)$. Extracellular vesicles (EVs) are considered as significant factors in inflammation and immune responses, antigen presentation, cancer progression and metastasis, immunomodulation, coagulation, tissue regeneration, organ repair, cell-cell communication, senescence, proliferation and differentiation, etc. in the body (25). Since they were discovered in the last decades of twentieth century, always there have been different opinions about their functions. Though EVs were initially known as cellular waste carriers, introducing fundamental features revealed that these nano/micro-sized particles play important biological roles in all aspects of life-from single cells to mammalians $(6,7)$. EV is a collective term which refers to heterogeneous cell-secreted structures comprised of a bilayer phospholipid membrane surrounding a wide range of macromolecules including proteins, lipids, and nucleic acids (e.g., cell specific antigens, surface markers, adhesion molecules, ligands, receptors, enzymes, miRNAs, IncRNAs, mRNAs, growth factors, etc.) $(5,8)$. According to parameters like their various biochemical, morphological, and biogenesis, EVs are classified into two groups; 
exosomes and ectosomes (9). The term 'exosome' was initially used for vesicles with sizes about 30$200 \mathrm{~nm}$ which were released from a wide range of cultured cells (i.e., their exact origin was not determined) and carrying 5 '-nucleotidase activity $(5,10)$. Currently, the term exosome is adopted to refer to intraluminal vesicles (ILVs) formed by reverse budding of endosomal membrane and finally secreted upon fusion of multivesicular endosomes (MVE) or late endosomes with plasma membrane $(2,9)$. On the other hand, ectosomes or microvesicles with sizes about $50-5000 \mathrm{~nm}$, are directly shed from plasma membrane and released into intercellular space (11).

To date, five different isolation methods of EVs including; ultracentrifugation-based methods, sizebased methods, Immunoaffinity capture-based methods, precipitation-based methods, and microfluidic-based methods have been developed (12) (An schematic illustration of the most prevalent methods for isolation of EVs, is shown in Figure 1). There are potential advantages and disadvantages for each method, which researchers should be considered in choosing an appropriate method based on their needs and possibilities. The ultracentrifugation-based method (UC) is a gold standard method in isolation of extracellular vesicles. In this method, a heterogeneous mixture is subjected to a centrifugal force and particles in the mixture can be separated according to their physical properties as well as density and viscosity of the solvent (12). There are more additional steps in order to obtain desired particles. In the first step, a low speed spin $(300 \times \mathrm{g})$ is needed to eliminate dead cells and bulky debris. After depletion of larger particles, $2,000 \times \mathrm{g}$ centrifugal force is applied to eliminate remaining cells and debris. In the next step, larger EVs like microvesicles are pelleted in the forces which varies among laboratories $(10,000$ $20,000 \times \mathrm{g})$. EVs are then pelleted at high speed spin $(100,000-120,000 \times \mathrm{g})(13,14)$. One drawback of ultracentrifugation is co-precipitation of protein aggregates, nucleosomal fragments and apoptotic bodies, which results in less purity of final extracts.
Therefore, often UC is used in combination with density gradient (DG) methods like sucrose density gradients or sucrose cushions which separate EVs according to their floatation densities (15). Using volume-excluding polymers like polyethylene glycol (PEG) is another option for isolation EVs from diverse biofluids. In this method, the precipitate can be isolated using low speed centrifugation or filtration. Polymer-based precipitation is an easy to use method, which does not need any requirements or specialized equipment (16), but there are some concerns about the purity of final extracts (14). In this short review, the intent is not to provide an extensive review about the isolation methods of EVs. Herein, promising breakthroughs about utilization of EVs in regenerative medicine are highlighted.

\section{Regenerative medicine}

Regenerative medicine is defined as various approaches and actions to replace lost tissue(s) with new tissues/cells or enhance regeneration of damaged tissues in a broad spectrum of indications (e.g., myocardial infraction, osteoarthritis, lung diseases, acute kidney injuries, chronic wounds, muscular dystrophies, bone and cartilage defects, etc.) $(4,5,17-19)$. There are different strategies towards tissue/organ regeneration, from cell transplantation to utilizing biomaterials alongside stem cell therapy, which are called tissue engineering (20). Despite stem cell transplantation is adopted as one of the major approaches in regenerative medicine, it exhibits some limitations. Transplanted cells might be tumorigenic in some cases or injected stem cells trapped in some organs (e.g., inside the lungs) (4). In the recent years, a promising strategy has been emerged, which facilitates regeneration process and hampers side effects of other approaches when used in combination with them (5). This cell-free based approach utilizes EVs to inspire cell therapist and tissue engineers to control immune reactions as well as regeneration at the same time. Regenerative properties of EVs are, at least in part, attributed to their protein and miRNA contents (20). 
120 Extracellular Vesicles in.....

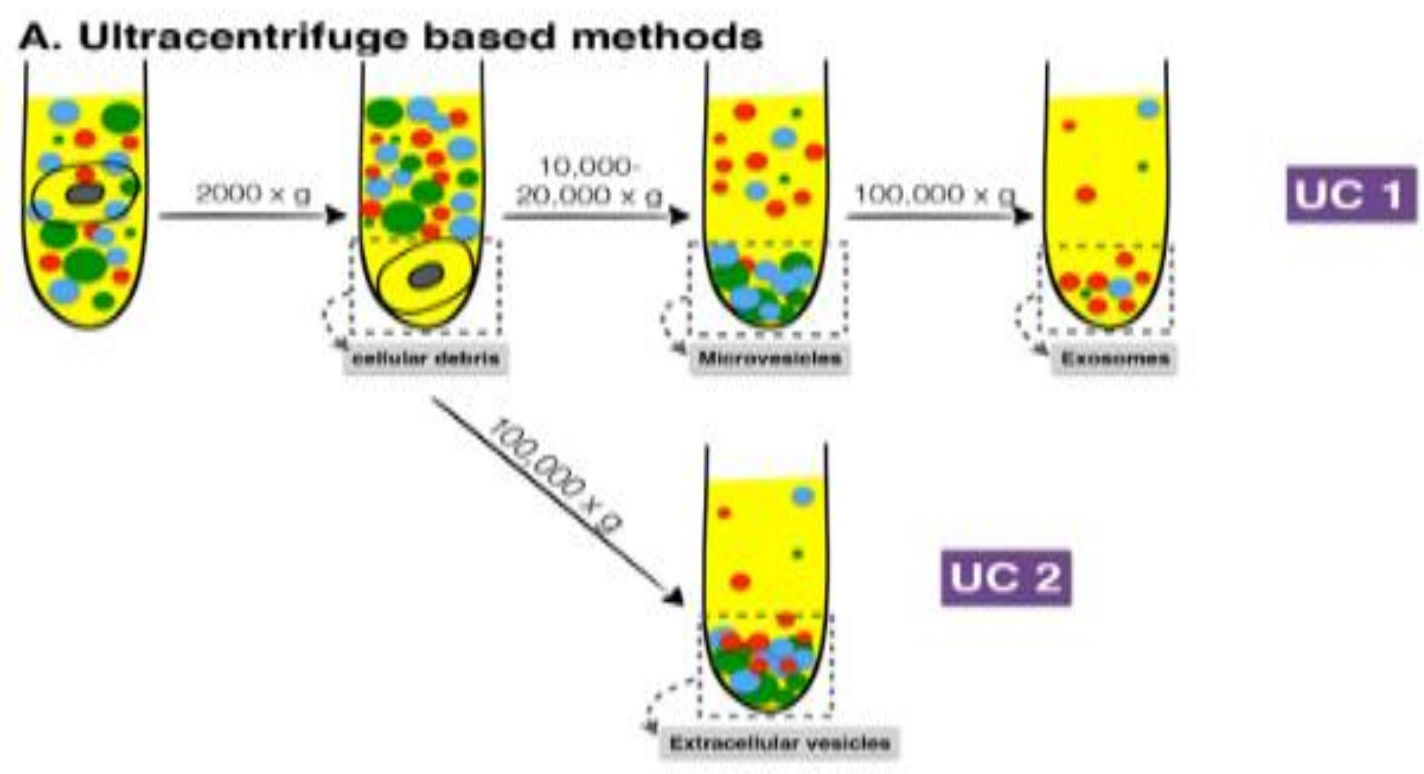

B. Sucrose density gradient based methods

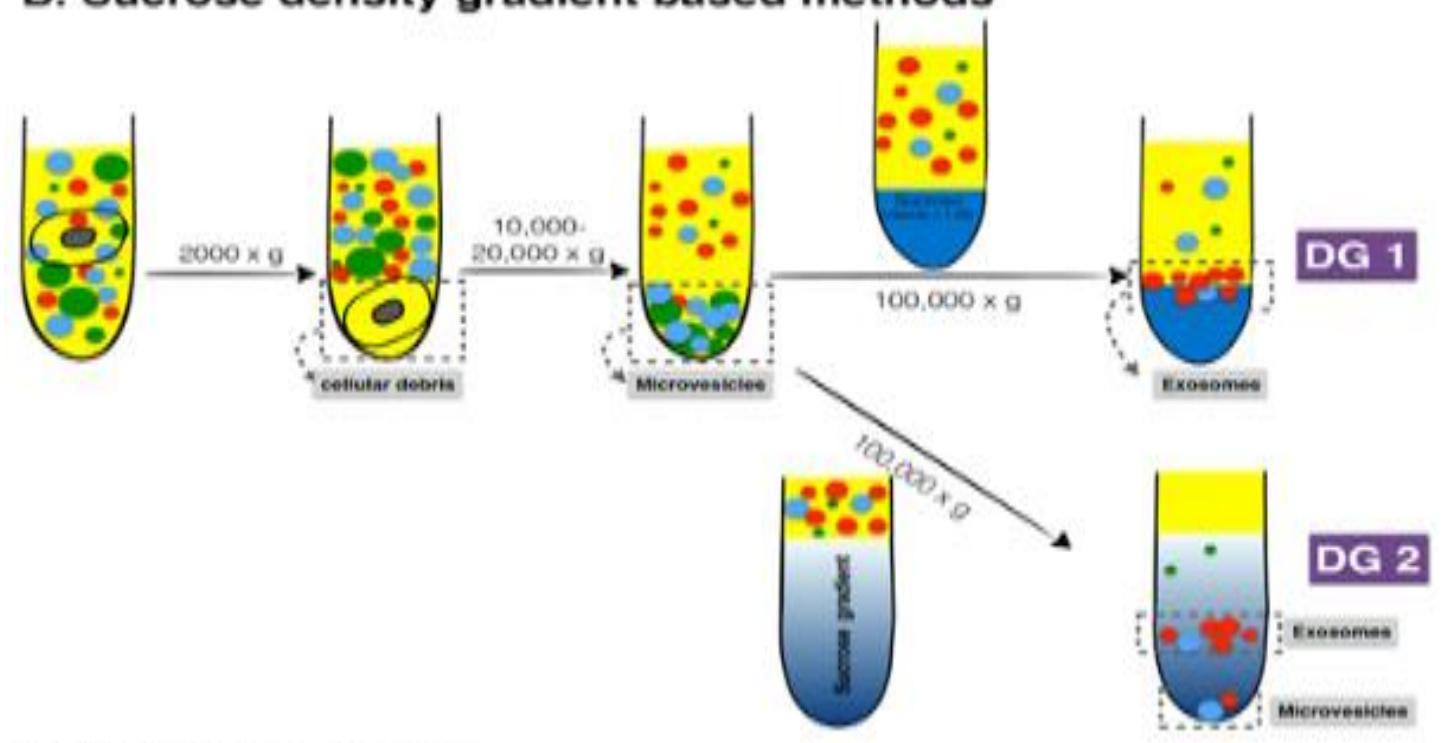

C. PEG precipitation

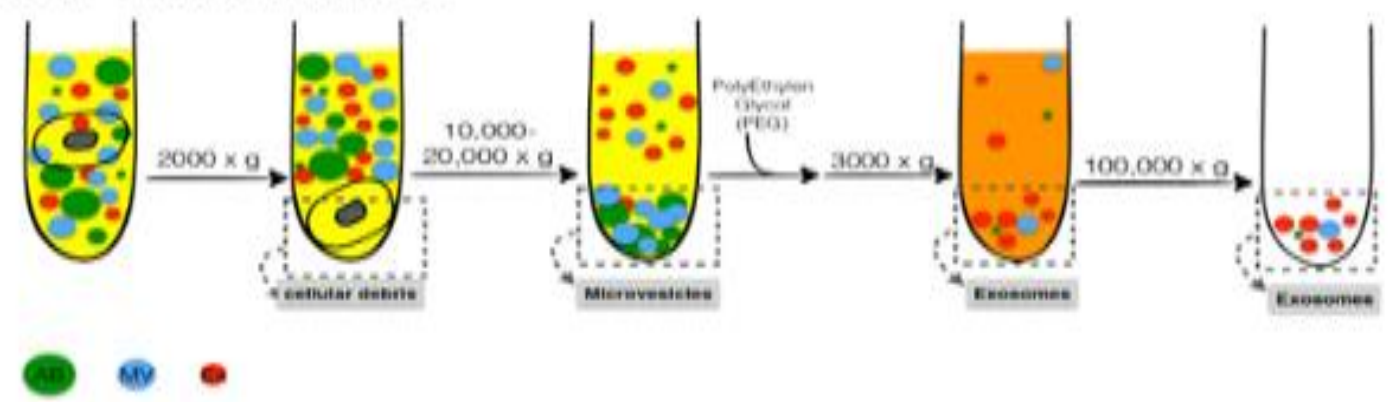

Fig1. Schematic illustration of the most prevalent methods for isolation of extracellular vesicles; A: Ultracentrifuge-based methods, B: Sucrose density gradient-based methods, C: PEG precipitation-based methods (AB: Apoptotic Bodies, Ex: Exosomes, DG: Density Gradient, MV: Microvesicles, UC: Ultracentrifuge, PEG: Poly-Ethylene Glycol). 


\section{Extracellular vesicles in regenerative medicine}

EVs derived from mesenchymal stem/stromal cells (MSCs) and other probable sources are one of the options on the table to regenerate and repair damaged or diseased tissues with lower risks and limitations (21). The investigations span a broad usage of EVs along with other materials (i.e., cells, tissues, biomaterials, etc.) or alone $(20,22)$. In the last few years, numerous studies have been carried out in order to reveal the potential roles of EVs, especially exosomes, in tissue repair and regeneration, but their potential roles have not been fully elucidated (21).

Zhang et al. proposed an approach to increase cutaneous wound healing due to collagen synthesis promotion based on exosomes secretion of humaninduced Pluripotent Stem Cell-derived MSCs (hiPSC-MSC-Exos). They found that proliferation and migration of human dermal fibroblasts as well as angiogenesis could be affected by concentration of exosomes secreted by hiPSC-MSC (23). Balbi et al. showed that $\mathrm{c}-\mathrm{KIT}^{+}$human amniotic fluid stem cells dynamically released EVs (hAFS-EVs) while the cells were under hypoxic conditions. hAFS-EVs represented remarkable regenerative and immunomodulatory effects on a model of skeletal muscle atrophy (HSA-Cre, $\mathrm{Smn}^{\mathrm{F} 7 / \mathrm{F} 7}$ mice) (24). Despite the fact that significant improvements have been made in regeneration of bone defects in recent years, there are still controversial issues, which shall be addressed. Innovative strategies using EVs have paved the way for new options in bone and cartilage regeneration. In a study carried out by Qi et al., in vitro experiments revealed that hiPSC-MSC-Exos could enhance cell proliferation, and alkaline phosphatase (ALP) activity. Also, mRNA upregulation besides increased protein expression of osteoblast-related genes were observed. On the other hand, in vivo studies showed that hiPSC-MSC-Exos dramatically altered bone regeneration and angiogenesis in critical-sized calvarial defects in ovariectomized rats (25). Liu et al. (26) investigated the effects of iPSC-MSC-Exos on osteonecrosis of the femoral head $(\mathrm{ONFH})$. Their results revealed an increase in proliferation, migration and tube-forming capacities of endothelial cells, whereas PI3K/Akt signaling pathway was activated by iPSC-MSCExos. As a matter of fact, considering newly proposed methods is an inevitable parameter in tissue/organ repair and regeneration. Therefore, a deep search is prerequisite for handling future studies. Table 1 illustrates some translational and in vivo studies employing cell/stem cell-derived microvesicles and exosomes in the case of regeneration and tissue repair in recent years.

\section{Concluding remarks and future prospects}

Regenerative medicine seeks the most applicable approaches to overcome some limitations in tissue remodeling, tissue regeneration and organ repair. The future medicine will be attributed with the most facile recovery methods in personalized medicine, which are compatible, economic and possess better regenerative effects. A major hurdle in the case of cell-based approaches toward regeneration is entrapment of injected cells inside some organs like lungs. These cells are somehow high-risked due to their tumorigenesis. Accordingly, EVs have been approved to be reliable substitutions instead of direct cell administration. It is still unclear which contents/properties of exosomes are capable of promoting tissue regeneration. At last but not least, by virtue of exosomal engineering, we might be able to modify the contents of exosomes by adding therapeutic drugs or compounds to enhance their regenerative potential in regenerative medicine. Moreover, seeking newly proposed strategies like combination therapies (i.e., Cell-therapy/EVs, small molecule treatments/EVs and Cell Therapy/small molecule treatments), will pave the way of a new era in the future regenerative medicine. 


\section{Extracellular Vesicles in.....}

Table 1. In vivo studies employing cell/stem cell-derived microvesicles and exosomes in the case of regeneration and tissue repair (Some parts are adopted from Ref. (18)

\begin{tabular}{|c|c|c|c|c|c|c|}
\hline Target tissue/Model & Cellular Source & $\begin{array}{c}\text { Species-exosome (Origin } \\
\text { into Target) }\end{array}$ & Cell-derived agent & Method & Dose & Reference \\
\hline Heart/IR & MSC & Human into Mouse & Exosomes & HPLC & $0.4 \mathrm{mg}$ & (27) \\
\hline Heart/infarct & MSC & Rat into Rat & Exosomes (w/GATA4) & ExoQuick & $\left(4 \times 10^{6} \mathrm{MSC}\right)$ & (28) \\
\hline Heart/IR & MSC & Human into Mouse & Exosomes, ATP & HPLC & $0.1-0.4 \mathrm{mg}$ & (29) \\
\hline Heart/infarct & MSC & Human into Rat & EVs & $100 \mathrm{~K} \times \mathrm{g}$ & $80 \mathrm{mg}$ & (30) \\
\hline Heart/infarct & MSC & Rat into Rat & Exosomes & ExoQuick & $80 \mathrm{mg}$ & (31) \\
\hline Kidney/gentamycin & MSC & Rat into Rat & Exosomes & $100 \mathrm{~K} \times \mathrm{g}$ & $100 \mathrm{mg}$ & (32) \\
\hline Kidney/cisplatin & MSC & Human into Rat & Exosomes & $100 \mathrm{~K} \times \mathrm{g}$ & $250 \mathrm{mg}$ & (33) \\
\hline Brain/TBI & MSC & Human into Rat & Exosomes & ExoQuick & $100 \mu \mathrm{g}$ & (34) \\
\hline Brain/TBI & MSC & Human into Mouse & Exosomes & An Chrom & $30 \mathrm{mg}$ & (35) \\
\hline Brain/stroke & MSC & Rat into Rat & Exosomes & $100 \mathrm{~K} \times \mathrm{g}$ & $100 \mathrm{mg}$ & (36) \\
\hline Brain/ischemia & MSC & Human into Ovine & EVs & PEG & $\left(1 \times 2 \times 10^{7} \mathrm{MSC}\right)$ & (37) \\
\hline Brain/TBI & MSC & Rat into Rat & Exosomes & ExoQuick & $100 \mathrm{mg}$ & (38) \\
\hline Brain/stroke & MSC & Human into Mouse & Exosomes & $110 \mathrm{~K} \times \mathrm{g}$ & $\left(2 \times 10^{6} \mathrm{MSCs}\right)$ & (39) \\
\hline Liver/fibrosis & MSC & Human into Rat & Exosomes & $100 \mathrm{~K} \times \mathrm{g}$ & $250 \mathrm{mg}$ & (40) \\
\hline Liver/drug injury & MSC & Human into Mouse & Exosomes & $100 \mathrm{~K} \times \mathrm{g}$ & $0.4 \mathrm{mg}$ & (41) \\
\hline Lung/hypoxia & MSC & Mouse into Mouse & Conditioned Medium, Exosomes & PEG-S200 & $0.1-10 \mathrm{mg}$ & (42) \\
\hline Lung/drug & MSC & Mouse into Mouse & Exosomes & $100 \mathrm{~K} \times \mathrm{g}$ & $25 \mathrm{mg}$ & (43) \\
\hline Lung/silicosis & MSC & Human into Mouse & Microvesicles & ExoQuick & $10 \mathrm{mg}$ & (44) \\
\hline Hypertension & MSC & Human into Mouse & Microvesicles & $100 \mathrm{~K} \times \mathrm{g}$ & $\left(3 \times 10^{6} \mathrm{MSCs}\right)$ & (45) \\
\hline Lung/fluid filled & MSC & Human into Human & Microvesicles & $100 \mathrm{~K} \times \mathrm{g}$ & $160 \mathrm{mg}$ & (46) \\
\hline Lung/E.coli endotoxin & MSC & Human into Mouse & Microvesicles & $100 \mathrm{~K} \times \mathrm{g}$ & $\left(9 \times 10^{6} \mathrm{MSCs}\right)$ & (47) \\
\hline Intestine/enterocolitis & MSC & Human into Rat & Exosomes & PureExo & $50 \mathrm{ml} \mathrm{IP}$ & (48) \\
\hline Intestine/enterocolitis & MSC & Rat into Rat & Microvesicles & $100 \mathrm{~K} \times \mathrm{g}$ & $50-200 \mathrm{mg}$ & (49) \\
\hline Skin/wound & MSC & Human into Rat & Exosomes, Wnt 4 & $100 \mathrm{~K} \times \mathrm{g}$ & $200 \mathrm{mg}$ & (50) \\
\hline Skin/wound & MSC & Human into Rat & Exosomes & $100 \mathrm{~K} \times \mathrm{g}$ & $160 \mathrm{mg}$ & (23) \\
\hline Skin/wound & MSC & Human into Mouse & Exosomes, miRNA & $120 \mathrm{~K} \times \mathrm{g}$ & $100 \mathrm{mg}$ & (51) \\
\hline Limb ischemia & MSC & Human into Mouse & Exosomes & $100 \mathrm{~K} \times \mathrm{g}$ & $200 \mathrm{mg}$ & (52) \\
\hline Skeletal Muscle/cardiotoxin & MSC & Human into Mouse & Exosomes, miR-494 & $110 \mathrm{~K} \times \mathrm{g}$ & $50 \mu 1$ & (53) \\
\hline Skeletal Muscle/ALS & MSC & Mouse into Mouse & Exosomes, SOD1 & PureExo & $0.2 \mathrm{mg} / \mathrm{ml}$ & (54) \\
\hline Wound healing in T2DM & nAT-MSCs & Human into Mouse & Microvesicles & $100 \mathrm{~K} \times \mathrm{g}$ & $\left(3 \times 10^{5} \mathrm{MSCs}\right)$ & (55) \\
\hline $\begin{array}{c}\text { Cisplatin and } \\
\text { Glycerol induced } \\
\text { AKI }\end{array}$ & hWJMSC & Human into Mouse & Microvesicles & $100 \mathrm{~K} \times \mathrm{g}$ & $100 \mu \mathrm{g}$ & (56) \\
\hline $\begin{array}{l}\text { Glycerol induced } \\
\text { AKI }\end{array}$ & MSC & Human into Mouse & Microvesicles & $100 \mathrm{~K} \times \mathrm{g}$ & $15 \mu \mathrm{g}$ & (57) \\
\hline $\begin{array}{l}\text { Liver/Model of } 70 \% \\
\text { hepatectomy }\end{array}$ & HLSCs & Human into Rat & Microvesicles & $100 \mathrm{~K} \times \mathrm{g}$ & $15 \mu \mathrm{g}$ & (58) \\
\hline Eye/ONC model & MSCs & Human into Rat & Exosomes & $100 \mathrm{~K} \times \mathrm{g}$ & $\left(1 \times 2 \times 10^{6} \mathrm{MSC}\right)$ & (59) \\
\hline $\begin{array}{l}\mathrm{SCI} / \text { model of rat cervical } \\
\text { avulsion }\end{array}$ & $\begin{array}{l}\text { Cortical neurons } \\
\text { treated with the RAR } \beta \\
\text { agonist CD2019 }\end{array}$ & Human into Rat & Exosomes & $100 \mathrm{~K} \times \mathrm{g}$ & $10 \mu \mathrm{g}$ & (60) \\
\hline Heart/MI & $\mathrm{CDCs}$ & Human into Mouse & Exosomes & ExoQuick & $3.5 \times 10^{8}$ or $2 \times 10^{8}$ & (61) \\
\hline $\begin{array}{l}\text { ischemia/reperfusion injury and } \\
\text { partial hepatectomy }\end{array}$ & hepatocytes & Mouse into Mouse & Exosomes & ExoQuick & $200 \mu \mathrm{g}$ & (62) \\
\hline Chronic cutaneous wounds & platelet-rich plasma & Human into Rat & Exosomes & $\begin{array}{c}100 \mathrm{~K} \times \mathrm{g}+30 \% \\
\text { sucrose- } \mathrm{D}_{2} \mathrm{O} \\
\text { cushion }\end{array}$ & $50 \mu \mathrm{g} / \mathrm{ml}$ & (63) \\
\hline Osteoarthritis & $\begin{array}{l}\text { human synovial } \\
\text { membrane MSCs / } \\
\text { iPSC-MSCs }\end{array}$ & Human into Mouse & Exosomes & Ultrafiltration & $10^{10} / \mathrm{ml}$ & (64) \\
\hline $\begin{array}{l}\text { Dental Pulp Tissue } \\
\text { Regeneration }\end{array}$ & $\begin{array}{l}\text { naïve human dental } \\
\text { pulp stem cells } \\
\text { (DPSCs) and human } \\
\text { bone marrow derived } \\
\text { stromal cells } \\
\text { (HMSCs) }\end{array}$ & Human into Mouse & Exosomes & ExoQuick & $\begin{array}{l}1.25 \times 10^{6} \mathrm{HMSCs}+ \\
250 \times 10^{3} \mathrm{DPSCs}\end{array}$ & (65) \\
\hline
\end{tabular}

\section{Abbreviations}

AKI: Acute Kidney Injury, ALS: Amyotrophic lateral sclerosis, CDCs: cardiosphere-derived cells, DPSCs: naïve human dental pulp stem cells, EVs: Extracellular Vesicles, HLSCs: Human Liver Stem Cells, hWJMSC-MVs: human Wharton-Jelly MSCs derived microvesicles, iPSC: induced Pluripotent Stem Cells, IR: myocardial ischemia/ reperfusion injury, HPLC: High Pressured Liquid Chromatography, PEG: Polyethylene Glycol, MI: Myocardial Infraction, miR: microRNA, MSC: Mesenchymal Stem/Stromal Cell, nAT-MSCs: non-diabetic healthy donor adipose tissue derived mesenchymal stem/stromal cells, ONC: Optic Nerve Crush, SCI: Spinal Cord Injuries, TBI: Traumatic Brain Injury, T2DM: Type II Diabetes Mellitus. 


\section{Conflict of Interest:}

Authors declared no conflict of interest.

\section{References}

1. Deatherage BL, Cookson BT. Membrane vesicle release in bacteria, eukaryotes, and archaea: a conserved yet underappreciated aspect of microbial life. Infect Immun. 2012;80(6):194857.

2. Colombo M, Raposo G, Théry C. Biogenesis, Secretion, and Intercellular Interactions of Exosomes and Other Extracellular Vesicles. Annu Rev Cell Dev Biol. 2014;30(1):255-89.

3. Bobrie A, Colombo M, Raposo G, Thery C. Exosome secretion: molecular mechanisms and roles in immune responses. Traffic. 2011;12(12):1659-68.

4. De Jong OG, Van Balkom BW, Schiffelers RM, Bouten CV, Verhaar MC. Extracellular vesicles: potential roles in regenerative medicine. Front Immunol. 2014;5:608.

5. Fuster-Matanzo A, Gessler F, Leonardi T, Iraci N, Pluchino S. Acellular approaches for regenerative medicine: on the verge of clinical trials with extracellular membrane vesicles? Stem Cell Res Ther. 2015;6:227.

6. Fu H, Hu D, Zhang L, Tang P. Role of extracellular vesicles in rheumatoid arthritis. Mol Immunol. 2018;93:125-32.

7. Robbins PD, Dorronsoro A, Booker CN. Regulation of chronic inflammatory and immune processes by extracellular vesicles. J Clin Invest. 2016;126(4):1173-80.

8. Robbins PD, Morelli AE. Regulation of immune responses by extracellular vesicles. Nat Rev Immunol. 2014;14(3):195-208.

9. van Niel G, D'Angelo G, Raposo G. Shedding light on the cell biology of extracellular vesicles. Nat Rev Mol Cell Biol. 2018;19(4): 213-28.

10. Trams EG, Lauter CJ, Salem JN, Heine U. Exfoliation of membrane ecto-enzymes in the form of micro-vesicles. Biochim Biophys Acta. 1981;645(1):63-70.

11. Cocucci E, Racchetti G, Meldolesi J. Shedding microvesicles: artefacts no more. Trends Cell Biol. 2009;19(2):43-51.

12. Li P, Kaslan M, Lee SH, Yao J, Gao Z. Progress in Exosome Isolation Techniques. Theranostics. 2017;7(3):789-804.
13. Momen-Heravi F, Balaj L, Alian S, Mantel PY, Halleck AE, Trachtenberg AJ, et al. Current methods for the isolation of extracellular vesicles. Biol Chem. 2013;394(10):1253-62.

14. Witwer KW, Buzas EI, Bemis LT, Bora A, Lasser C, Lotvall J, et al. Standardization of sample collection, isolation and analysis methods in extracellular vesicle research. J Extracell Vesicles. 2013;2.

15. Zeringer E, Barta T, Li M, Vlassov AV. Strategies for isolation of exosomes. Cold Spring Harb Protoc. 2015;2015(4):319-23.

16. Batrakova EV, Kim MS. Using exosomes, naturally-equipped nanocarriers, for drug delivery. J Control Release. 2015;219:396-405.

17. Shabbir A, Cox A, Rodriguez-Menocal L, Salgado M, Van Badiavas E. Mesenchymal Stem Cell Exosomes Induce Proliferation and Migration of Normal and Chronic Wound Fibroblasts, and Enhance Angiogenesis In Vitro. Stem Cells Dev. 2015;24(14):1635-47.

18. Phinney DG, Pittenger MF. Concise Review: MSC-Derived Exosomes for Cell-Free Therapy. Stem Cells. 2017;35(4):851-8.

19. Zhang S, Chu WC, Lai RC, Lim SK, Hui JH, Toh WS. Exosomes derived from human embryonic mesenchymal stem cells promote osteochondral regeneration. Osteoarthritis Cartilage. 2016;24(12):2135-40.

20. Bjorge IM, Kim SY, Mano JF, Kalionis B, Chrzanowski W. Extracellular vesicles, exosomes and shedding vesicles in regenerative medicine a new paradigm for tissue repair. Biomater Sci. 2017;6(1):60-78.

21. Jing $\mathrm{H}, \mathrm{He} \mathrm{X}$, Zheng J. Exosomes and regenerative medicine: state of the art and perspectives. Transl Res. 2018;196:1-16.

22. Riazifar M, Pone EJ, Lotvall J, Zhao W. Stem Cell Extracellular Vesicles: Extended Messages of Regeneration. Annu Rev Pharmacol Toxicol. 2017;57:125-54.

23. Zhang J, Guan J, Niu X, Hu G, Guo S, Li Q, et al. Exosomes released from human induced pluripotent stem cells-derived MSCs facilitate cutaneous wound healing by promoting collagen synthesis and angiogenesis. J Transl Med. 2015;13:49. 
24. Balbi C, Piccoli M, Barile L, Papait A, Armirotti A, Principi E, et al. First Characterization of Human Amniotic Fluid Stem Cell Extracellular Vesicles as a Powerful Paracrine Tool Endowed with Regenerative Potential. Stem Cells Transl Med. 2017;6(5):1340-55.

25. Qi X, Zhang J, Yuan H, Xu Z, Li Q, Niu X, et al. Exosomes Secreted by Human-Induced Pluripotent Stem Cell-Derived Mesenchymal Stem Cells Repair Critical-Sized Bone Defects through Enhanced Angiogenesis and Osteogenesis in Osteoporotic Rats. Int J Biol Sci. 2016;12(7):836-49.

26. Liu X, Li Q, Niu X, Hu B, Chen S, Song W, et al. Exosomes Secreted from Human-Induced Pluripotent Stem Cell-Derived Mesenchymal Stem Cells Prevent Osteonecrosis of the Femoral Head by Promoting Angiogenesis. Int J Biol Sci. 2017;13(2):232-44.

27. Lai RC, Arslan F, Lee MM, Sze NS, Choo A, Chen TS, et al. Exosome secreted by MSC reduces myocardial ischemia/reperfusion injury. Stem Cell Res. 2010;4(3):214-22.

28. Yu B, Kim HW, Gong M, Wang J, Millard RW, Wang Y, et al. Exosomes secreted from GATA-4 overexpressing mesenchymal stem cells serve as a reservoir of anti-apoptotic microRNAs for cardioprotection. Int J Cardiol. 2015;182:349-60.

29. Arslan F, Lai RC, Smeets MB, Akeroyd L, Choo A, Aguor EN, et al. Mesenchymal stem cellderived exosomes increase ATP levels, decrease oxidative stress and activate PI3K/Akt pathway to enhance myocardial viability and prevent adverse remodeling after myocardial ischemia/reperfusion injury. Stem Cell Res. 2013;10(3):301-12.

30. Bian S, Zhang L, Duan L, Wang X, Min Y, Yu H. Extracellular vesicles derived from human bone marrow mesenchymal stem cells promote angiogenesis in a rat myocardial infarction model. J Mol Med (Berl). 2014;92(4):387-97.

31. Teng X, Chen L, Chen W, Yang J, Yang Z, Shen Z. Mesenchymal Stem Cell-Derived Exosomes Improve the Microenvironment of Infarcted Myocardium Contributing to Angiogenesis and Anti-Inflammation. Cell Physiol Biochem. 2015;37(6):2415-24.

32. Reis LA, Borges FT, Simões MJ, Borges AA, Sinigaglia-Coimbra R, Schor N. Bone MarrowDerived Mesenchymal Stem Cells Repaired but
Did Not Prevent Gentamicin-Induced Acute Kidney Injury through Paracrine Effects in Rats. PLoS ONE. 2012;7(9):e44092.

33. Zhou Y, Xu H, Xu W, Wang B, Wu H, Tao Y, et al. Exosomes released by human umbilical cord mesenchymal stem cells protect against cisplatininduced renal oxidative stress and apoptosis in vivo and in vitro. Stem Cell Res Ther. 2013;4(2):34.

34. Zhang Y, Chopp M, Zhang ZG, Katakowski M, Xin H, Qu C, et al. Systemic administration of cell-free exosomes generated by human bone marrow derived mesenchymal stem cells cultured under 2D and 3D conditions improves functional recovery in rats after traumatic brain injury. Neurochem Int. 2017;111:69-81.

35. Kim DK, Nishida H, An SY, Shetty AK, Bartosh TJ, Prockop DJ. Chromatographically isolated CD63+CD81+ extracellular vesicles from mesenchymal stromal cells rescue cognitive impairments after TBI. Proc Natl Acad Sci U S A. 2016;113(1):170-5.

36. Xin H, Li Y, Cui Y, Yang JJ, Zhang ZG, Chopp M. Systemic administration of exosomes released from mesenchymal stromal cells promote functional recovery and neurovascular plasticity after stroke in rats. J Cereb Blood Flow Metab. 2013;33(11):1711-5.

37. Ophelders DR, Wolfs TG, Jellema RK, Zwanenburg A, Andriessen P, Delhaas T, et al. Mesenchymal Stromal Cell-Derived Extracellular Vesicles Protect the Fetal Brain After HypoxiaIschemia. Stem Cells Transl Med. 2016;5(6):75463.

38. Zhang Y, Chopp M, Meng Y, Katakowski M, Xin $\mathrm{H}$, Mahmood A, et al. Effect of exosomes derived from multipluripotent mesenchymal stromal cells on functional recovery and neurovascular plasticity in rats after traumatic brain injury. $\mathbf{J}$ Neurosurg. 2015;122(4):856-67.

39. Doeppner TR, Herz J, Gorgens A, Schlechter J, Ludwig AK, Radtke S, et al. Extracellular Vesicles Improve Post-Stroke Neuroregeneration and Prevent Postischemic Immunosuppression. Stem Cells Transl Med. 2015;4(10):1131-43.

40. Li T, Yan Y, Wang B, Qian H, Zhang X, Shen L, et al. Exosomes derived from human umbilical cord mesenchymal stem cells alleviate liver fibrosis. Stem Cells Dev. 2013;22(6):845-54. 
41. Tan CY, Lai RC, Wong W, Dan YY, Lim SK, Ho HK. Mesenchymal stem cell-derived exosomes promote hepatic regeneration in drug-induced liver injury models. Stem Cell Res Ther. 2014;5(3):76

42. Lee C, Mitsialis SA, Aslam M, Vitali SH, Vergadi E, Konstantinou G, et al. Exosomes mediate the cytoprotective action of mesenchymal stromal cells on hypoxia-induced pulmonary hypertension. Circulation. 2012;126(22):260111.

43. Aliotta JM, Pereira M, Wen S, Dooner MS, Del Tatto M, Papa E, et al. Exosomes induce and reverse monocrotaline-induced pulmonary hypertension in mice. Cardiovasc Res. 2016;110(3):319-30.

44. Choi M, Ban T, Rhim T. Therapeutic use of stem cell transplantation for cell replacement or cytoprotective effect of microvesicle released from mesenchymal stem cell. Mol Cells. 2014;37(2):133-9.

45. Zhu YG, Feng XM, Abbott J, Fang XH, Hao Q, Monsel A, et al. Human mesenchymal stem cell microvesicles for treatment of Escherichia coli endotoxin-induced acute lung injury in mice. Stem Cells. 2014;32(1):116-25.

46. Gennai S, Monsel A, Hao Q, Park J, Matthay MA, Lee JW. Microvesicles Derived From Human Mesenchymal Stem Cells Restore Alveolar Fluid Clearance in Human Lungs Rejected for Transplantation. Am J Transplant. 2015;15(9):2404-12.

47. Monsel A, Zhu YG, Gennai S, Hao Q, Hu S, Rouby JJ, et al. Therapeutic Effects of Human Mesenchymal Stem Cell-derived Microvesicles in Severe Pneumonia in Mice. Am J Respir Crit Care Med. 2015;192(3):324-36

48. Rager TM, Olson JK, Zhou Y, Wang Y, Besner GE. Exosomes secreted from bone marrowderived mesenchymal stem cells protect the intestines from experimental necrotizing enterocolitis. J Pediatr Surg. 2016;51(6):942-7.

49. Yang J, Liu XX, Fan H, Tang Q, Shou ZX, Zuo $\mathrm{DM}$, et al. Extracellular Vesicles Derived from Bone Marrow Mesenchymal Stem Cells Protect against Experimental Colitis via Attenuating Colon Inflammation, Oxidative Stress and Apoptosis. PLoS One. 2015;10(10):e0140551.
50. Zhang B, Wang M, Gong A, Zhang X, Wu X, Zhu $\mathrm{Y}$, et al. HucMSC-Exosome Mediated-Wnt4 Signaling Is Required for Cutaneous Wound Healing. Stem Cells. 2015;33(7):2158-68

51. Fang S, Xu C, Zhang Y, Xue C, Yang C, Bi H, et al. Umbilical Cord-Derived Mesenchymal Stem Cell-Derived Exosomal MicroRNAs Suppress Myofibroblast Differentiation by Inhibiting the Transforming Growth Factor-beta/SMAD2 Pathway During Wound Healing. Stem Cells Transl Med. 2016;5(10):1425-39.

52. Hu GW, Li Q, Niu X, Hu B, Liu J, Zhou SM, et al. Exosomes secreted by human-induced pluripotent stem cell-derived mesenchymal stem cells attenuate limb ischemia by promoting angiogenesis in mice. Stem Cell Res Ther. 2015;6:10

53. Nakamura Y, Miyaki S, Ishitobi H, Matsuyama S, Nakasa T, Kamei N, et al. Mesenchymal-stemcell-derived exosomes accelerate skeletal muscle regeneration. FEBS Lett. 2015;589(11):1257-65.

54. Bonafede R, Scambi I, Peroni D, Potrich V, Boschi F, Benati D, et al. Exosome derived from murine adipose-derived stromal cells: Neuroprotective effect on in vitro model of amyotrophic lateral sclerosis. Exp Cell Res. 2016;340(1):150-8.

55. Trinh NT, Yamashita T, Tu TC, Kato T, Ohneda $\mathrm{K}$, Sato F, et al. Microvesicles enhance the mobility of human diabetic adipose tissue-derived mesenchymal stem cells in vitro and improve wound healing in vivo. Biochem Biophys Res Commun. 2016;473(4):1111-8.

56. Zou X, Zhang G, Cheng Z, Yin D, Du T, Ju G, et al. Microvesicles derived from human Wharton's Jelly mesenchymal stromal cells ameliorate renal ischemia-reperfusion injury in rats by suppressing CX3CL1. Stem Cell Res Ther. 2014;5(2):40.

57. Bruno S, Grange C, Deregibus MC, Calogero RA, Saviozzi S, Collino F, et al. Mesenchymal stem cell-derived microvesicles protect against acute tubular injury. J Am Soc Nephrol. 2009;20(5):1053-67.

58. Herrera MB, Fonsato V, Gatti S, Deregibus MC, Sordi A, Cantarella D, et al. Human liver stem cell-derived microvesicles accelerate hepatic regeneration in hepatectomized rats. J Cell Mol Med. 2010;14(6B):1605-18. 
59. Mead B, Tomarev S. Bone Marrow-Derived Mesenchymal Stem Cells-Derived Exosomes Promote Survival of Retinal Ganglion Cells Through miRNA-Dependent Mechanisms. Stem Cells Transl Med. 2017;6(4):1273-85.

60. Goncalves MB, Malmqvist T, Clarke E, Hubens CJ, Grist J, Hobbs C, et al. Neuronal RARbeta Signaling Modulates PTEN Activity Directly in Neurons and via Exosome Transfer in Astrocytes to Prevent Glial Scar Formation and Induce Spinal Cord Regeneration. J Neurosci. 2015;35(47):15731-45.

61. Ibrahim AG, Cheng K, Marban E. Exosomes as critical agents of cardiac regeneration triggered by cell therapy. Stem Cell Reports. 2014;2(5):60619.

62. Nojima H, Freeman CM, Schuster RM, Japtok L, Kleuser B, Edwards MJ, et al. Hepatocyte exosomes mediate liver repair and regeneration via sphingosine-1-phosphate. J Hepatol. 2016;64(1):60-8.

63. Guo SC, Tao SC, Yin WJ, Qi X, Yuan T, Zhang $\mathrm{CQ}$. Exosomes derived from platelet-rich plasma promote the re-epithelization of chronic cutaneous wounds via activation of YAP in a diabetic rat model. Theranostics. 2017;7(1):8196.

64. Zhu Y, Wang Y, Zhao B, Niu X, Hu B, Li Q, et al. Comparison of exosomes secreted by induced pluripotent stem cell-derived mesenchymal stem cells and synovial membrane-derived mesenchymal stem cells for the treatment of osteoarthritis. Stem Cell Res Ther. 2017;8(1):64.

65. Huang CC, Narayanan R, Alapati S, Ravindran S. Exosomes as biomimetic tools for stem cell differentiation: Applications in dental pulp tissue regeneration. Biomaterials. 2016;111:103-15.

\section{How to Cite This Article:}

Soleymani-Goloujeh M, Saberi S, Shekari F. Extracellular Vesicles in Regenerative Medicine, a Brief Review. Mod Med Lab J. 2019; 2 (2) :118-126 